\title{
Effect of gestational age on the morphometric parameters of placenta
}

\author{
Nazeefa HMFJ ${ }^{1}$, Thirukumar $\mathrm{M}^{2}$, Thayabaran $\mathrm{M}^{1}$ \\ ${ }^{I}$ Department of Human Biology, Faculty of Health-Care Sciences, Eastern University, \\ Sri Lanka. \\ ${ }^{2}$ Department of Clinical Sciences, Faculty of Health-Care Sciences, Eastern University, \\ Sri Lanka. \\ *nazeefaj@esn.ac.lk
}

Placenta plays a vital role in the foetal growth and development. Alteration in its morphological parameters is associated with adverse pregnancy outcome. Hence, in the present study the influence of gestational age on the weight, thickness and number of cotyledon formation of the placenta were assessed. The study was conducted on 289 singleton normal vaginal deliveries in Teaching Hospital, Batticaloa. The gestational age was determined from last menstrual period and confirmed by early ultrasonography. The placentae were washed to remove the blood clots and the weight and thickness were measured. The number of cotyledons was counted through careful examination. The collected data were analysed by Spearman's correlation test using SPSS software. The gestational age exhibited a positive relationship with the weight of the placenta $(p=0.007)$. However, the correlation coefficient (0.176) was very weak according to Evans (1996) suggestion. The thickness and number of cotyledons showed significant relationship ( $\mathrm{p}=0.000)$ with the weight of the placentae than gestational age. The weight of the foetus increases with the weight of the placenta. This indicates that placental growth restriction leads to restriction in the intrauterine growth of foetus. The study result shows that advance gestational age support the growth and development of the placenta. However, the maternal factors such as nutritional values of the diet, BMI, gestational weight gain and pregnancy induced conditions might influence the weight of the placenta. Therefore, the effects of maternal factors on the placental weight need to be studied. 